\title{
Spontaneous Spectral Diffusion in CdSe Quantum Dots
}

\author{
Mark J. Fernée, ${ }^{* \dagger, \ddagger, \nabla}$ Taras Plakhotnik, ${ }^{\S, \nabla}$ Yann Louyer, ${ }^{\perp, \nabla}$ Bradley N. Littleton, ${ }^{\#, \nabla}$ Christian Potzner,,$\|, \nabla$ \\ Philippe Tamarat, ${ }^{\dagger \ddagger, \nabla}$ Paul Mulvaney, $\|, \nabla$ and Brahim Lounis ${ }^{\dagger, \ddagger, \nabla}$ \\ †Université Bordeaux, LP2N, F-33405 Talence, France \\ ${ }^{*}$ CNRS \& Institut d'Optique, LP2N, F-33405 Talence, France \\ ${ }^{\S}$ School of Physical Sciences, The University of Queensland, Queensland 4072, Australia \\ ${ }^{\perp}$ Université Bordeaux, LOMA, F-33405 Talence, France \\ \#Department of Physics, King's College London, Strand, London, WC2R 2LS United Kingdom \\ "School of Chemistry, The University of Melbourne, Parkville, Victoria 3010, Australia
}

\section{Supporting Information}

ABSTRACT: Spectral diffusion of the emission line of single colloidal nanocrystals is generally regarded as a random process. Here, we show that each new spectral position has a finite memory of previous spectral positions, as evidenced by persistent anticorrelations in time series of spectral jumps. The anticorrelation indicates that there is an enhanced probability of the charge distribution around the nanocrystal returning to a previous configuration. We show both statistically and directly that this memory manifests as an observable spontaneous "relaxation" in the absence of a pump laser, so that spectral diffusion progresses in a manner of "two steps forward and one step back".

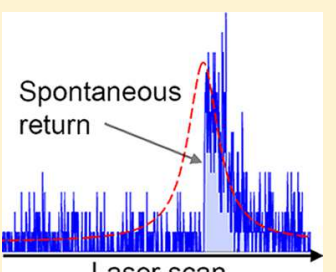

SECTION: Physical Processes in Nanomaterials and Nanostructures

ingle nanocrystal quantum dots (NCs) have been $\checkmark$ developed for a wide range of applications, from biological labeling to the light-absorbing materials in the next generation of photovoltaics. However, their adoption in certain applications has been hampered by a number of idiosyncrasies, photoluminescence blinking and spectral diffusion (i.e., environmentally induced fluctuation in the emission energy of individual quantum emitters). ${ }^{1,2}$ In particular, photoluminescence blinking has received a considerable amount of attention ${ }^{3}$ as it strongly impacts applications involving single NCs. The ultimate aim is to understand and, through NC engineering, 4 mitigate blinking. Until recently, spectral diffusion (SD) had received far less attention, and therefore, there is less understanding of the underlying photophysical properties of this phenomenon. At low temperatures, NCs have narrow spectral lines, ${ }^{6-9}$ which could be exploited for quantum optical applications, ${ }^{10}$ but SD will be detrimental to such applications.

In colloidal NCs, spectral diffusion results from electric field fluctuations in the local environment of the NC that induces a shift of the band edge spectra due to the Stark effect. ${ }^{11}$ The fundamental cause of SD remains uncertain. Two distinct time scales for SD have been identified, a SD process that occurs on the microsecond time scale that results in broadening of the spectral line that is only observable using various highresolution spectroscopies ${ }^{12}$ and a SD process that occurs at second time scales that results in discrete jumps of the spectral position. ${ }^{13}$ This latter SD process is the most commonly studied and the most disruptive process due to the size of the spectral jumps and is the focus of this work. The spectral jumps have been attributed to individual charges moving around the surface, ${ }^{14,15}$ although this attribution is merely speculative. For example, scanning electron force microscopy has shown that many, but not all, NCs have or develop surface charges, ${ }^{16}$ while $\mathrm{SD}$ is a ubiquitous phenomenon observed in all single NC studies. In addition, SD can be seen to persist through transitions between neutral and charged states, ${ }^{7}$ suggesting that a free surface charge is not responsible. In general, the surface of a NC is a complex interface between crystal surface atoms and molecular ligands that bind to individual surface sites in order to passivate dangling bonds. It is known that the surface coverage is not complete; ${ }^{17}$ thus, a NC has many possible surface configurations, which in turn have different associated charge distributions. Thus, a reasonable model of SD is one involving photoactivated rearrangement of surface ligands causing discrete jumps ${ }^{13}$ between different surface configurations in a complex potential landscape. ${ }^{18}$ Such an interpretation is compatible with the $\mathrm{SD}$ model originally proposed by Empedocles and Bawendi. ${ }^{14}$

Early reports of SD in NCs revealed a thermal activation energy below which the SD process is solely photoactivated. ${ }^{14,15}$ This motivates the study of SD at cryogenic temperatures, where competing thermal effects are eliminated, effectively isolating the photophysical properties. A recent numerical study of the response of surface ligands to photoactivation has revealed that they can "jump" between different lattice sites. ${ }^{19}$ Such dynamics could also admit the possibility of spontaneous spectral diffusion for surface ligands promoted to an unstable higher-energy configuration can relax

Received: April 16, 2012

Accepted: June 6, 2012 
back to a more stable lower-energy configuration. Spontaneous $\mathrm{SD}$ is known in the field of single-molecule spectroscopy, where polymer matrixes are known to not be in a state of thermal equilibrium, allowing SD of a molecular transition to occur in the absence of photophysical pumping. ${ }^{20}$ The ligands that passivate the surface of a NC have much in common with random polymer matrixes, and therefore, we may expect similar behavior with SD in NCs. Therefore, testing for spontaneous SD will give further insight into the physical origin of SD and ultimately aid the design of new materials that mitigate this effect.

In this Letter, we use cryogenic photoluminescence spectroscopy along with high-resolution resonant photoluminescence excitation spectroscopy (RPLE) to probe the origins of $\mathrm{SD}$. An autocorrelation analysis of time series of spectral jumps is used to test the randomness of the SD phenomenon. We then test for spontaneous SD by comparing statistics of a spectral jump distribution obtained with continuous excitation with a spectral jump distribution comprising spectral shifts following brief periods with no pump laser. We also employ a complementary technique of RPLE of the band edge exciton state using a scanning single-frequency laser that can cause photoinduced jumps and detect any spontaneous return.

For statistical analysis, a time series of spectral peak positions is generated using a peak fitting routine to determine the positions of emission maxima in single NC spectra. A typical time series of spectral peak positions determined from a succession of single NC spectra obtained at cryogenic temperatures (here $5 \mathrm{~K}$ ) is shown in Figure 1a. The time

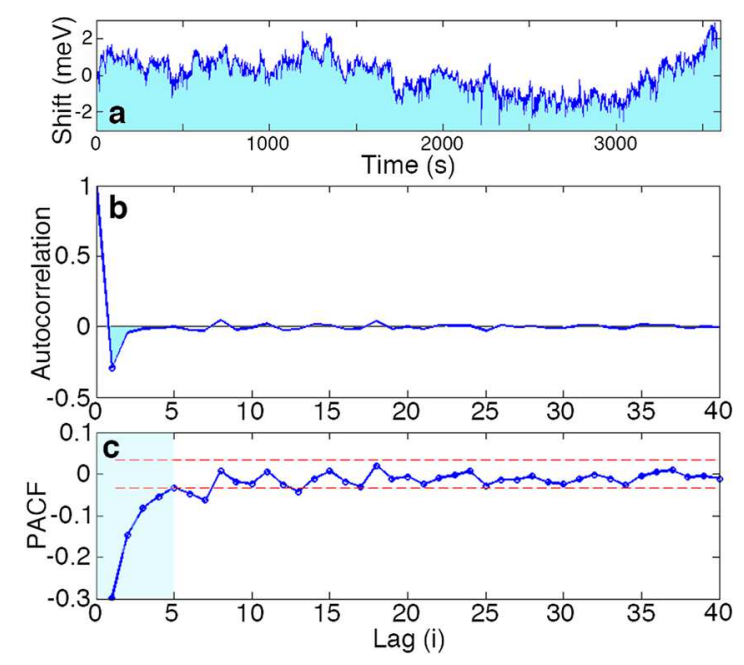

Figure 1. (a) Time series of spectral peak positions obtained with a pump irradiance of $52 \mathrm{~W} / \mathrm{cm}^{2}$ and a $1 \mathrm{~s}$ integration at $5 \mathrm{~K}$. (b) Autocorrelation of the time series of spectral jumps calculated from the peak position time series in (a). (c) Partial autocorrelation calculated from the time series of spectral jumps, with $95 \%$ confidence limits indicated (red dashed lines). The shaded region indicates the extent of the anticorrelation.

series appears to exhibit random walk-like behavior. This is borne out when we take the autocorrelation of the time series and see an apparent exponential decay, characteristic of a random walk (not shown). However, if we calculate the autocorrelation of the spectral jump time series (obtained as the series of successive difference between neighboring spectral positions), we see in Figure $1 \mathrm{~b}$ that there is a pronounced anticorrelation at the first delay (lag 1). This indicates that, after a spectral jump, it is more likely for the next jump to return the emission to the previous position. Thus, the spectral jumps are not truly random but have some finite memory of previous configurations. The depth of this memory is tested using the partial autocorrelation of the spectral jump series, as shown in Figure 1c. The partial autocorrelation is a technique commonly used to determine the number of terms that need to be used in an autoregression model, $\operatorname{AR}(k),{ }^{21}$ of the form, $x_{n}=\sum_{i=1}^{k} a_{i} x_{n-i}$ $+\varepsilon$, where $k$ is the order of the model, $\varepsilon$ is a normally distributed white noise term, and the $a_{i}$ are the model coefficients and represent a memory of previous values in the series. From the partial autocorrelation, we see that this depth of memory could be satisfied by an $\mathrm{AR}(5)$ model, indicating that the memory vanishes after approximately five spectral acquisitions.

We have recently reported correlations in SD time series that were related to Poissonian jump statistics. ${ }^{13}$ In this case, the correlations were very sensitive to the experimental integration time and were easily lost. However, the anticorrelations reported in this Letter are relatively insensitive to the integration time and pump power. The robustness of the anticorrelations to variations in integration time and pump power is reflected in the partial autocorrelation result of Figure 1c, which gives an indication of the depth of the memory. We have checked this memory by increasing the integration time and find that the anticorrelations do indeed persist in accordance with the partial autocorrelation function. Furthermore, similar anticorrelations were found for most spectral time series (including data presented in refs 13 and 18; see Supporting Information Table S1), suggesting these anticorrelations to be a general characteristic of SD.

Monte Carlo simulations of the SD process were conducted in order to test the previously reported power-law-distributed two-level system (TLS) model of SD. ${ }^{18}$ In general, such models were only able to reproduce the anticorrelations by modifying the power law at high frequencies. Such a modification has no impact on the previously reported power law as the highfrequency region has little effect on those statistics (see Supporting Information Figure S1). Nevertheless, the observation of a robust anticorrelation in SD time series indicates a departure from the pure power law behavior previously reported. ${ }^{18}$

The anticorrelations revealed in Figure 1 indicate that spectral jumps are not purely random but retain a memory of past charge configurations. In particular, it is reasonable to assume a competition between the photophysical process driving the $\mathrm{SD}$ and a relaxation process toward a previous charge configuration. In order to test the possibility that the relaxation corresponds to spontaneous $\mathrm{SD}$, we generate statistics for spectral jumps occurring across extended periods where the excitation laser is blocked, so-called dark jumps. This is conducted using a rotating beam block that introduces periods with no pump (ie. dark periods) interleaved with bright periods, as shown in Figure 2a. The statistics on the dark jumps are then compiled by calculating the difference between the last spectral peak position before a dark period and the first spectral position measured following a dark period. The periods of continuous laser excitation (ie. bright periods) were long enough to ensure a significant change in the local charge distribution (as indicated by significant SD in Figure 2a). Thus, there can be no memory of previous dark periods. Evidence for spontaneous spectral jumps will manifest in the dark jump distribution deviating from the bright jump distribution. In 

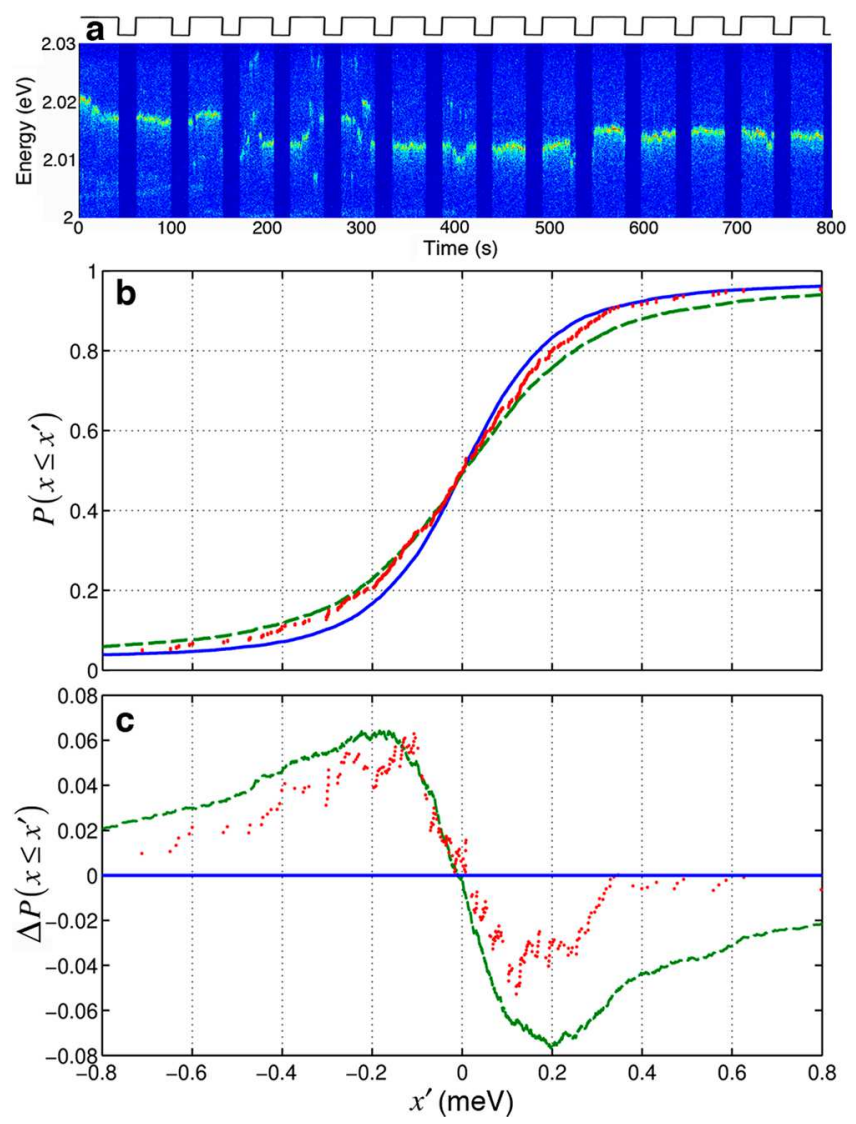

Figure 2. (a) Time series of spectra (vertical axis) obtained at $5 \mathrm{~K}$ with a pump irradiance of $340 \mathrm{~W} / \mathrm{cm}^{2}$ and an integration time of $1 \mathrm{~s}$. During the acquisition, the pump laser was periodically blocked with $\sim 35 \mathrm{~s}$ of laser on and $\sim 18 \mathrm{~s}$ of laser off to introduce dark periods into the time series. (b) Cumulative distributions obtained from an ensemble of seven concatenated single $\mathrm{NC}$ data at $5 \mathrm{~K}$, corresponding to 11746 spectral jumps and 292 dark jumps. The $2 I$ (blue solid), $3 I$ (green dashed), and dark jumps data are indicated (red dots). (c) Deviations of the $3 I$ (green dashed) and dark jump (red dots) CDFs from the 2I CDF (solid blue line).

order to quantify this deviation, we compute two bright jump distributions $^{13,18}$ corresponding to the difference between successive spectral peak positions (ie. $j_{i}=x_{i}-x_{i-1}$ ), called the $2 I$ distribution and the $3 I$ distribution, corresponding to the jumps calculated according to $j_{i}=x_{i}-x_{i-2}$ (indicating an additional period of illumination). These two SD distributions simply correspond to different illumination periods, where $I$ represents the illumination period required to acquire a single spectrum. Resolving a spectral jump requires a minimum of two spectrum acquisitions, or $2 I$. Thus, the $3 I$ jump distribution will be broader than the $2 I$ jump distribution due to SD induced during the additional illumination period, and therefore, a dark jump distribution broader than the $2 I$ distribution would indicate the presence of spontaneous SD.

In Figure $2 b$, we plot the cumulative distribution functions (CDFs) for $2 I$ and $3 I$. The CDF is another form of the probability distribution that for some value, $x^{\prime}$, gives the probability $P\left(x \leq x^{\prime}\right)$. Thus, the CDF can be described in terms of the traditional probability distribution function as follows, $P\left(x \leq x^{\prime}\right)=\int_{-\infty}^{x \prime} P(x) \mathrm{d} x$. CDFs are used here as they allow all of the data to be represented as individual points without the need for binning into histograms, which can be misleading. This is especially useful for data sets with a small number of points, such as the dark jump data. The CDF is obtained from a concatenated time series of seven different NCs obtained under identical conditions. In order to highlight the dark jump behavior, we have also plotted the deviation away from the $2 I$ CDF for both the $3 I$ and dark jump CDFs in Figure 2c. Here, the dark jump CDF clearly matches the 3I CDF near the center of the distribution, but it more closely matches the $2 I$ distribution at the extremities. This provides clear evidence for spectral jumps occurring during dark periods without laser illumination, although the extent to which this dark SD occurs is clearly limited. These data categorize the dark jumps into two energy regimes: Spectral jumps larger than $\sim 0.4 \mathrm{meV}$ more closely follow the $2 I$ distribution, indicating that these jumps are likely the result of irreversible charge redistributions (that may relax on longer time scales), while jumps smaller than $\sim 0.15 \mathrm{meV}$, which follow the $3 I$ distribution, are characteristic of reversible charge redistributions that spontaneously relax to a prior charge distribution.

We also employed resonant excitation of a NC using a singlefrequency dye laser to scan through the spectral line while detecting the Stokes shifted emission in the LO phonon replica (ie. $\mathrm{RPLE}^{6}$ ). This technique is complementary to the nonresonant excitation scheme used above in that the lack of signal indicates that the laser is not exciting the NC and hence represents a dark period. The technique can resolve single NC spectral lines with high resolution, as shown in Figure $3 \mathrm{~b}$. The stability of the NC emission is indicated in Figure 3c, representing an average of 18 scans ( $120 \mu \mathrm{eV}$ range) with only a small increase in line width. However, if we slow the scan

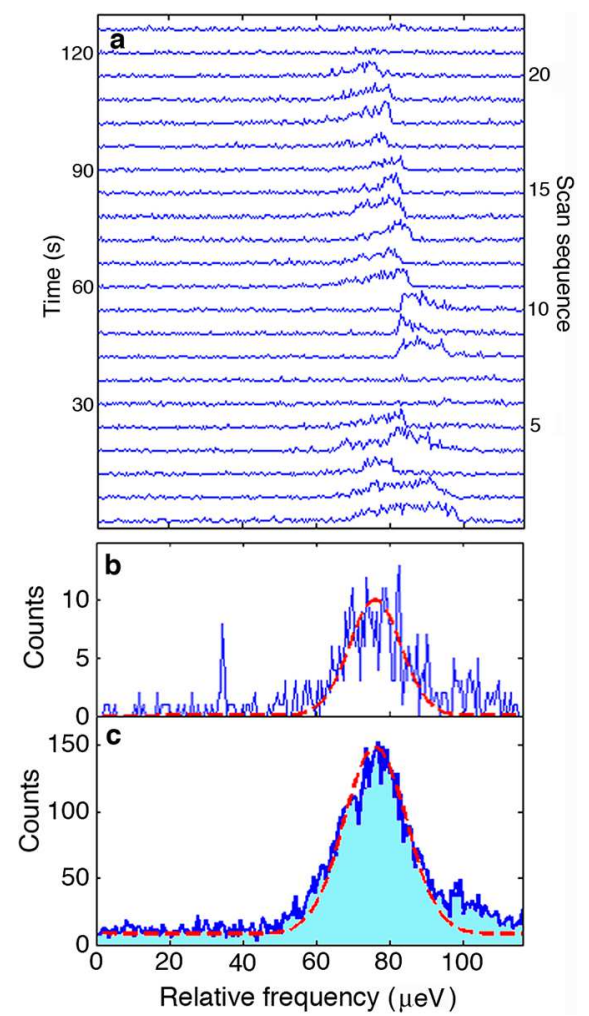

Figure 3. Resonant PLE scans at $2 \mathrm{~K}$ with a pump irradiance of 100 $\mathrm{W} / \mathrm{cm}^{2}$. (a) Series of successive slow scans (scan rate of $24 \mu \mathrm{eV} / \mathrm{s}$ ). (b) Single fast scan (scan rate of $120 \mu \mathrm{eV} / \mathrm{s}$ ) with a $16 \mu \mathrm{eV}$ Gaussian fit (red dashed). (c) Average of 18 successive fast scans with a $20 \mu \mathrm{eV}$ Gaussian fit (red dashed). 
rate by a factor of 5 , the NC is far less stable. In Figure 3a, we show a sequence of successive spectral scans obtained immediately after the faster scans used for Figure 3c. Under these conditions, each spectral position in successive scans is revisited every $6 \mathrm{~s}$, which accounts for the scan and return times. The sequence of spectra in Figure 3a is remarkable as it shows a NC that undergoes large photoinduced spectral jumps to positions outside of the scan range but then returns. These returns occur spontaneously, without any pump laser influence. Although one or two such events may be possible with an unobserved pump influence (ie. near the edge of the line, where the count rate falls into the noise), the sequence of 10 successive scans showing this behavior rules out a statistical anomaly. Furthermore, there are clear periods where the spectral line has vanished only to reappear a number of scans hence. This behavior is consistent with the statistical behaviors shown in Figures 1 and 2 and is an unambiguous example of spontaneous SD.

The observation of a spontaneous SD at temperatures as low as $2 \mathrm{~K}$ indicates that the process must either have a low activation energy or involve some alternative temperatureindependent mechanism. We speculate on the mechanism for spontaneous SD in the Supporting Information (section 3), invoking a model compatible with that originally proposed by Empedocles and Bawendi ${ }^{14}$ based on highly asymmetric surface configuration potentials. However, we note here that spontaneous SD is observed from single molecules embedded in molecular glasses ${ }^{20}$ at temperatures below $1 \mathrm{~K}$. Given the complexity of the surface topology and $\mathrm{NC} /$ surface-ligand interface, it is reasonable to speculate that processes present in molecular glasses may also be relevant to NCs, such as tunneling between nearly energy degenerate surface configurations.

In conclusion, we have reported a hitherto unexpected memory effect in SD, which manifests as a robust anticorrelation in a time series of spectral jumps. This memory is attributed to relaxation to a low-energy surface charge configuration. The possibility of spontaneous relaxation was tested statistically by introducing periods where the pump laser was blocked. The dark jump distributions were consistent with limited spectral evolution due to spontaneous SD. Direct detection of this spontaneous SD was accomplished using resonant excitation scans of the NC spectral line, where following spectral jumps out of the scan range, the spectral line subsequently returned in successive scans. Thus, spontaneous $\mathrm{SD}$ is consistent with the relaxation to a surface electronic configuration of lower energy, which indicates that the phenomenon of SD is actually a progression though a series of stable and unstable surface charge distributions. This highlights new surface dynamics and hints at the effect that phonons have on surface ligands. Further insight into the SD process will require more advanced strategies beyond pure optical spectroscopic studies, such as correlating multiple nanoscale probes. ${ }^{22}$ Ultimately, a greater understanding of the NC surface is crucial for understanding effects such as luminescence blinking and for engineering improvements to both photophysical and photochemical stability in these materials.

\section{EXPERIMENTAL METHODS}

The CdSe/CdZnS nanocrystals used in this study were either sourced from a commercial supplier (Invitrogen ITK605 and ITK655 with core radii of approximately 2.4 and $4.1 \mathrm{~nm}$, respectively $^{23}$ ) or were synthesized for this study as a CdSe core with a $\mathrm{CdS} / \mathrm{ZnS}$ shell, as previously reported. ${ }^{13,18}$ Experiments using the conventional spectroscopy have been previously described, including sample preparation. ${ }^{13}$ In order to collect the statistics for the dark jumps, a rotating shutter was employed, which was operated asynchronously with the acquisition. For the RPLE experiment, the samples (ITK655 NCs streptavidin conjugates) were spin-coated onto glass coverslips in a polyvinyl alcohol matrix, which helps stabilize the NCs for resonant excitation. The sample was mounted in a helium bath cryostat capable of achieving temperatures as low as $2 \mathrm{~K}$. For the RPLE measurements, a narrow band dye laser $(\sim 4 \mathrm{neV})$ was scanned across the NC resonance, and the optical phonon replica was detected with an avalanche photodiode, using a low-pass filter to remove the laser scatter. ${ }^{6}$

\section{ASSOCIATED CONTENT}

\section{S Supporting Information}

Anticorrelation data, Monte Carlo simulations, and asymmetric TLS potentials and spontaneous SD. This material is available free of charge via the Internet at http://pubs.acs.org.

\section{AUTHOR INFORMATION}

\section{Corresponding Author}

*E-mail: mjfernee@gmail.com.

\section{Author Contributions}

$\nabla_{\text {The }}$ manuscript was written through contributions of all authors. All authors have given approval to the final version of the manuscript. These authors contributed equally.

\section{Notes}

The authors declare no competing financial interest.

\section{ACKNOWLEDGMENTS}

M.J.F. wishes to thank John Fernée for his continued support and encouragement.

\section{ABBREVIATIONS}

$\mathrm{CDF}$, cumulative distribution function; NC, nanocrystal; RPLE, resonant photoluminescence excitation spectroscopy; SD, spectral diffusion

\section{REFERENCES}

(1) Nirmal, M.; Dabbousi, B.; Bawendi, M.; Macklin, J.; Trautman, J.; Harris, T.; Brus, L. Fluorescence Intermittency in Single Cadmium Selenide Nanocrystals. Nature 1996, 383, 802-804.

(2) Empedocles, S. A.; Norris, D. J.; Bawendi, M. G. Photoluminescence Spectroscopy of Single CdSe Nanocrystallite Quantum Dots. Phys. Rev. Lett. 1996, 77, 3873.

(3) Frantsuzov, P.; Kuno, M.; Janko, B.; Marcus, R. A. Universal Emission Intermittency in Quantum Dots, Nanorods and Nanowires. Nat. Phys. 2008, 4, 519.

(4) Hohng, S.; Ha, T. Near-Complete Suppression of Quantum Dot Blinking in Ambient Conditions. J. Am. Chem. Soc. 2004, 126, 1324-5.

(5) Wang, X.; Ren, X.; Kahen, K.; Hahn, M.; Rajeswaran, M.; Maccagnano-Zacher, S.; Silcox, J.; Cragg, G.; Efros, A.; Krauss, T. Non-Blinking Semiconductor Nanocrystals. Nature 2009, 459, 686689.

(6) Biadala, L.; Louyer, Y.; Tamarat, Ph.; Lounis, B. Direct Observation of the Two Lowest Exciton Zero-Phonon Lines in Single CdSe/ZnS Nanocrystals. Phys. Rev. Lett. 2009, 103, 037404.

(7) Fernée, M. J.; Littleton, B. N.; Rubinsztein-Dunlop, H. Detection of Bright Trion states using the Fine Structure Fingerprint of Single CdSe/CdZnS Colloidal Quantum Dots. ACS Nano 2009, 3, 3762. 
(8) Biadala, L.; Louyer, Y.; Tamarat, Ph.; Lounis, B. Band-Edge Exciton Fine Structure of Single CdSe/ZnS Nanocrystals in External Magnetic Fields. Phys. Rev. Lett. 2010, 105, 157402.

(9) Louyer, Y.; Biadala, L.; Trebbia, J.-B.; Fernée, M. J.; Tamarat, Ph.; Lounis, B. Efficient Biexciton Emission in Elongated CdSe/ZnS Nanocrystals. Nano Lett. 2011, 11, 4370-4375.

(10) Press, D.; Ladd, T. D.; Zhang, B.; Yamamoto, Y. Complete Quantum Control of a Single Quantum Dot Spin using Ultrafast Optical Pulses. Nature 2008, 456, 218-221.

(11) Empedocles, S. A.; Bawendi, M. G. Quantum-Confined Stark Effect in Single CdSe Nanocrystallite Quantum Dots. Science 1997, 278, 2114-7.

(12) Coolen, L.; Brokmann, X.; Spinicelli, P.; Hermier, J.-P. Emission Characterization of a Single CdSe-ZnS Nanocrystal with High Temporal and Spectral Resolution by Photon-Correlation Fourier Spectroscopy. Phys. Rev. Lett. 2008, 100, 027403.

(13) Fernée, M. j.; Littleton, B. N.; Plakhotnik, T.; RubinszteinDunlop, H.; Gómez, D.; Mulvaney, P. Charge Hopping Revealed by Jitter Correlations in the Photoluminescence Spectra of Single CdSe Nanocrystals. Phys. Rev. B 2010, 81, 155307.

(14) Empedocles, S. A.; Bawendi, M. G. Influence of Spectral Diffusion on the Line Shapes of Single CdSe Nanocrystallite Quantum Dots. J. Phys. Chem. B 1999, 103, 1826-30.

(15) Muller, J.; Lupton, J. M.; Rogach, A. L.; Feldmann, J.; Talapin, D. V.; Weller, H. Monitoring Surface Charge Migration in the Spectral Dynamics of Single CdSe/CdS Nanodot/Nanorod Heterostructures. Phys. Rev. B 2005, 72, 205339.

(16) Krauss, T. D.; O’Brien, S.; Brus, L. E. Charge and Photoionization Properties of Single Semiconductor Nanocrystals. J. Phys. Chem. B 2001, 105, 1725-1733.

(17) Jones, M.; Lo, S.; Scholes, G. D. Quantitative Modeling of the Role of Surface Traps in CdSe/CdS/ZnS Nanocrystal Photoluminescence Decay Dynamics. Proc. Natl. Acad. Sci. U.S.A. 2009, 106, 3011-3016.

(18) Plakhotnik, T.; Fernée, M. J.; Littleton, B. N.; RubinszteinDunlop, H.; Potzner, C.; Mulvaney, P. Anomalous Power-Law Statistics in Spectral Diffusion of Semiconductor Quantum Dots. Phys. Rev. Lett. 2010, 105, 167402.

(19) Voznyy, O. Mobile Surface Traps in CdSe Nanocrystals with Carboxylic Acid Ligands. J. Phys. Chem. C 2011, 115, 15927-15932.

(20) Boiron, A.-M.; Tamarat, Ph.; Lounis, B.; Brown, R.; Orrit, M. Are the Spectral Trails of Single Molecules Consistent with the Standard Two-Level System Model of Glasses at Low Temperatures? Chem. Phys. 1999, 247, 119-132.

(21) Brockwell, P. Davis, R. Time Series: Theory and Methods, 2nd ed.; Springer: New York, 1991.

(22) Vanmaekelbergh, D.; Casavola, M. Single-Dot Microscopy and Spectroscopy for Comprehensive Study of Colloidal Nanocrystals. J. Phys. Chem. Lett. 2011, 2, 2024.

(23) Liptay, T. J.; Marshall, L. F.; Rao, P. S.; Ram, R. J.; Bawendi, M. G. Anomalous Stokes Shift in CdSe Nanocrystals. Phys. Rev. B 2007, $76,155314$. 